\title{
Risk factors for ocular toxoplasmosis in Brazil
}

\author{
A. I. C. FERREIRA ${ }^{1} \dagger$, C. C. BRANDÃO DE MATTOS $^{1} \dagger$, F. B. FREDERICO ${ }^{2} \dagger$, \\ C.S. MEIRA ${ }^{3}$, G. C. ALMEIDA JR. ${ }^{2}$, F. NAKASHIMA ${ }^{1}$, C. R. BERNARDO ${ }^{1}$, \\ V. L. PEREIRA-CHIOCCOLA ${ }^{3}$ AND L. C. DE $\operatorname{MATTOS}^{1 *}$ \\ ${ }^{1}$ Immunogenetics Laboratory, Molecular Biology Department, Faculdade de Medicina de São José do Rio Preto \\ (FAMERP), São José do Rio Preto, SP, Brazil \\ ${ }^{2}$ Outpatient Eye Clinic, Fundação Faculdade Regional de Medicina-Hospital de Base (HB-FUNFARME), \\ São José do Rio Preto, SP, Brazil \\ ${ }^{3}$ Parasitology Laboratory, Instituto Adolfo Lutz, São Paulo, SP, Brazil
}

Received 30 September 2012; Final revision 5 February 2013; Accepted 10 February 2013; first published online 18 March 2013

\section{SUMMARY}

The aim of this study was to investigate risk factors for ocular toxoplasmosis (OT) in patients who received medical attention at a public health service. Three hundred and forty-nine consecutive patients, treated in the Outpatient Eye Clinic of Hospital de Base, São José do Rio Preto, São Paulo state, Brazil, were enrolled in this study. After an eye examination, enzyme-linked immunosorbent assay (ELISA) was used to determine anti-Toxoplasma gondii antibodies. The results showed that $25.5 \%$ of the patients were seronegative and $74.5 \%$ were seropositive for IgG anti-T. gondii antibodies; of these $27 \cdot 3 \%$ had OT and $72 \cdot 7 \%$ had other ocular diseases (OOD). The presence of cats or dogs [odds ratio (OR) 2.22, 95\% confidence interval (CI) 1.24-3.98, $P=0.009$ ] and consumption of raw or undercooked meat (OR 1.77, 95\% CI 1.05-2.98, $P=0.03$ ) were associated with infection but not with the development of OT. Age (OT 48.2 $\pm 21 \cdot 2$ years vs. OOD: $69 \cdot 5 \pm 14 \cdot 7$ years, $P<0 \cdot 0001)$ and the low level of schooling/literacy (OT vs. OOD: OR 0.414, 95\% CI 0.2231-0.7692, $P=0 \cdot 007$ ) were associated with OT. The presence of dogs and cats as well as eating raw/undercooked meat increases the risk of infection, but is not associated with the development of OT.

Key words: Eye infection, ocular toxoplasmosis, risk factors, Toxoplasma gondii, toxoplasmic retinochoroiditis, toxoplasmosis.

\section{INTRODUCTION}

Toxoplasmosis, a disease resulting from infection by the protozoan, Toxoplasma gondii, can be congenital

\footnotetext{
* Author for correspondence: Professor Dr L. C. de Mattos, Laboratório de Imunogenética, Departamento de Biologia Molecular, Faculdade de Medicina de São José do Rio Preto (FAMERP), Avenida Brigadeiro Faria Lima, 5416, 15090-000 São José do Rio Preto, SP, Brasil.

(Email: luiz.carlos@famerp.br)

$\dagger$ These authors contributed equally as joint first authors.
}

or acquired [1]. T. gondii is a versatile parasite for which the prevalence of infection varies between countries, geographical areas and ethnic groups living within a specific region [2]. In Brazil, infection rates range from $50 \%$ to $83 \%$ of the population depending on the location of the study [3]; the high prevalence worldwide is correlated to environmental risk factors and socioeconomic factors $[3,4]$.

There are several transmission routes with a large proportion of acquired infections related to the 
ingestion of cysts in raw or undercooked meat and contact with sporulated oocysts in the environment $[3,5]$.

Manifestations of infection by $T$. gondii are often subclinical, i.e. asymptomatic, or with non-specific symptoms [6]. Ocular toxoplasmosis (OT), associated with both congenital and acquired infections, is one of the most common manifestations of the disease $[3,7]$. OT is the main cause of posterior uveitis, a disease that can cause serious sequelae including complete loss of vision [8].

The prevalence of OT varies in different regions around the world; in Brazil about 30\% of patients with eye diseases have OT $[3,9,10]$. Several studies have investigated risk factors for congenital toxoplasmosis [11, 12]; however, few studies have evaluated factors related to the occurrence, severity and recurrence of OT.

The aim of this study was to investigate risk factors contributing to infection by $T$. gondii and to the development of OT in patients who received medical attention in an outpatient eye clinic of a tertiary teaching hospital of a public health service.

\section{MATERIALS AND METHODS}

\section{Patient selection}

Over a 2-year period (2009-2010), 349 consecutive male and female patients treated in the Outpatient Eye Clinic of Hospital de Base, Fundação Faculdade Regional de Medicina (HB-FUNFARME), a public health service in São José do Rio Preto, São Paulo state, Brazil were enrolled in this study. Each participant signed an informed consent form after receiving details about the objectives of the study and the procedures involved. This study was approved by the Research Ethics Committee of the Medical School in São José do Rio Preto, Brazil (FAMERP; no. 050/2009 dated 9 March 2009).

\section{Epidemiological data}

Participants completed a questionnaire on epidemiological data related to $T$. gondii infection, including social, environmental and economic factors and housing.

\section{Diagnosis of OT}

The clinical evaluation of patients was conducted by two experienced physicians (F.B.F. and G.C.A.Jr.), using an indirect binocular ophthalmoscope (Binocular Ophthalmoscope ID10, Topcon Corporation, USA). Subsequently, patients were allocated to either the OT group or the other ocular diseases (OOD) group.

\section{Blood sampling}

Serum, obtained for each patient from a peripheral blood sample collected without anticoagulant by venepuncture, was stored at $-20^{\circ} \mathrm{C}$ until use.

\section{Identification of IgG class anti-T. gondii antibodies}

After an eye examination, IgG class anti- $T$. gondii antibodies were investigated by enzyme-linked immunosorbent assay (ELISA) as described previously [13].

\section{Statistical analysis}

The results were analysed using GraphPad version 3.1 software (GraphPad Software Inc., USA). Fisher's exact test, odds ratio (OR) and $\chi^{2}$ test were used to compare independence between proportions, and ages were compared using Student's $t$ test. Differences were considered statistically significant at $P \leqslant 0 \cdot 05$.

\section{RESULTS}

The mean age of the 349 patients in this study was $56 \cdot 9 \pm 17 \cdot 0$ years (median 61 , minimum 18 , maximum 88 years); $187(53.6 \%)$ were male and $16(46.4 \%)$ were female. Ninety-eight percent $(n=342)$ resided in São Paulo state; $15 \cdot 5 \%(n=53)$ in São José do Rio Preto and $84.5 \%(n=289)$ in neighbouring towns. Four patients $(1 \cdot 1 \%)$ resided in the state of Minas Gerais and three $(0.9 \%)$ in the state of Mato Grosso do Sul. Based on the data, $73 \cdot 4 \%(n=256)$ owned a house and only $0 \cdot 3 \%(n=1)$ lived in wooden shacks.

Table 1 lists the socioeconomic parameters and environmental risk factors of patients who were seronegative and seropositive for $\operatorname{IgG}$ anti-T. gondii antibodies.

Table 2 shows the socioeconomic parameters and environmental risk factors of seropositive patients with OT and with OOD.

\section{DISCUSSION}

This study investigated the risk factors for $T$. gondii infection and for the development of OT. Several 
Table 1. Socioeconomic parameters and environmental risk factors of patients who are seropositive or seronegative for IgG anti-Toxoplasma gondii antibodies in an outpatient eye clinic, São José do Rio Preto, São Paulo state, Brazil*

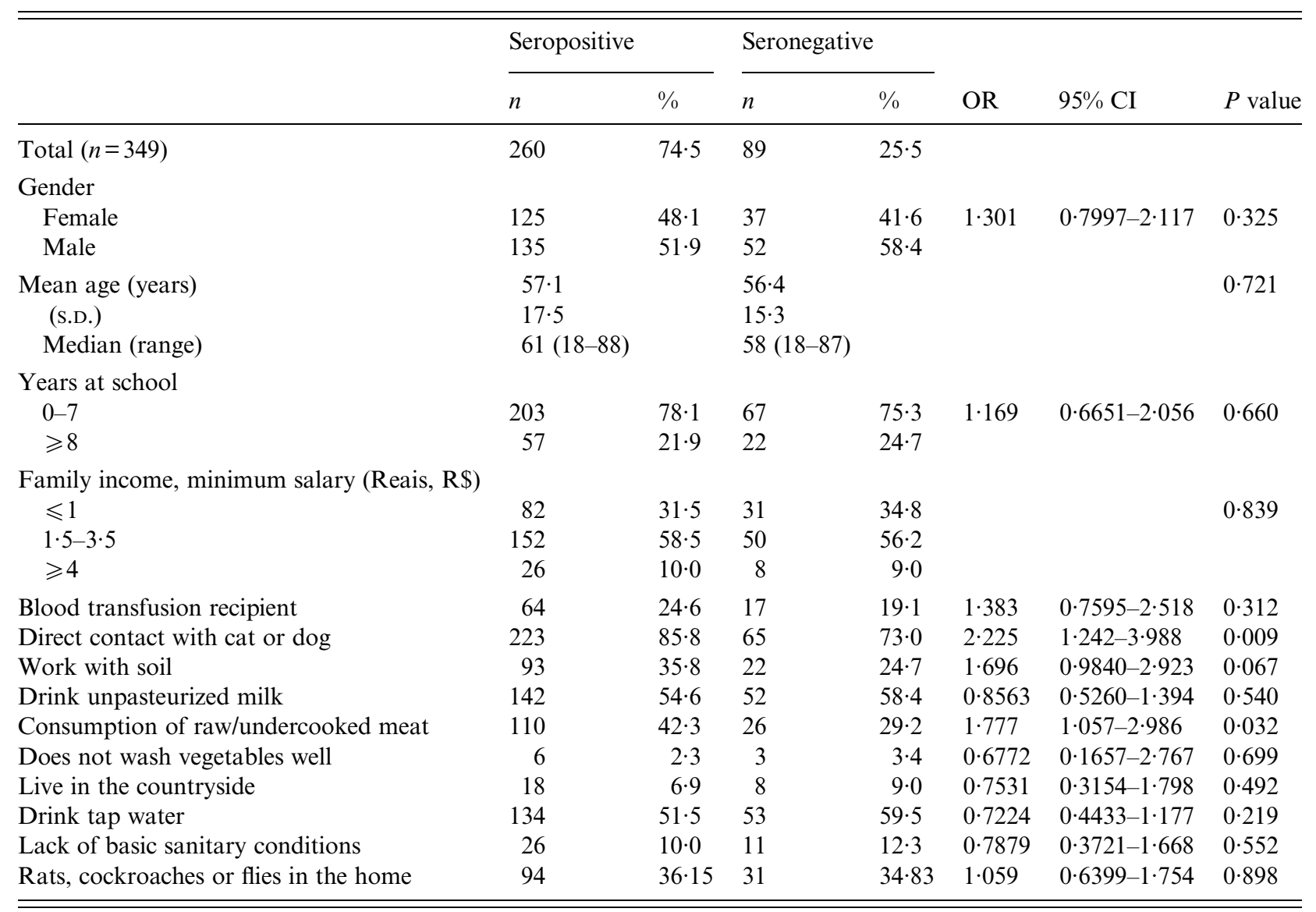

OR, Odds ratio; CI, confidence interval.

* Univariate analysis; $95 \%$ confidence interval, $P \leqslant 0 \cdot 05$.

studies have been conducted to ascertain the risk factors contributing to $T$. gondii infection in pregnant women [14-16]; however, there are few studies in outpatient eye clinics that address risk factors, in particular in patients who developed OT.

ELISA was used to detect IgG anti- $T$. gondii antibodies as recommended, as it is frequently used to determine whether an individual has anti- $T$. gondii antibodies [3, 17].

Patients were examined for the presence of ocular lesions by ophthalmoscopy. The high rate of patients infected by $T$. gondii in this study is in agreement with the literature reporting that this percentage can be as high as $80 \%$ in Brazil [3], including the region of this study [18-20].

The frequency of OT in seropositive patients demonstrates that eye diseases caused by $T$. gondii are common in individuals with ocular diseases in the study region. Dubey et al. report a lower rate of ocular lesions suggestive of toxoplasmosis than that found in the present study [3]. Thus the results reported here are not consistent with the findings of Dubey et al., probably due to differences in the sample population and the study region.

Indeed, the prevalence of ocular disease caused by T. gondii in Brazil varies greatly from $1 \cdot 1 \%$ to $27 \cdot 3 \%$ depending on the region but mainly the study population (Table 3). Most research was conducted as population-based studies, whereas in the current study the patients were selected in a referral eye clinic. Similar rates were reported by Gouveia et al. [28] in a study at a centre specializing in uveitis care in São Paulo city.

The parameters of age, gender, schooling and family income were evaluated in relation to the risk of infection and the development of OT. Comparing the two groups, no statistically significant differences were found between women and men. 
Table 2. Socioeconomic parameters and environmental risk factors of patients seropositive for anti-Toxoplasma gondii IgG antibodies with ocular toxoplasmosis (OT) or other ocular diseases (OOD), in an outpatient eye clinic, São José do Rio Preto, São Paulo state, Brazil*

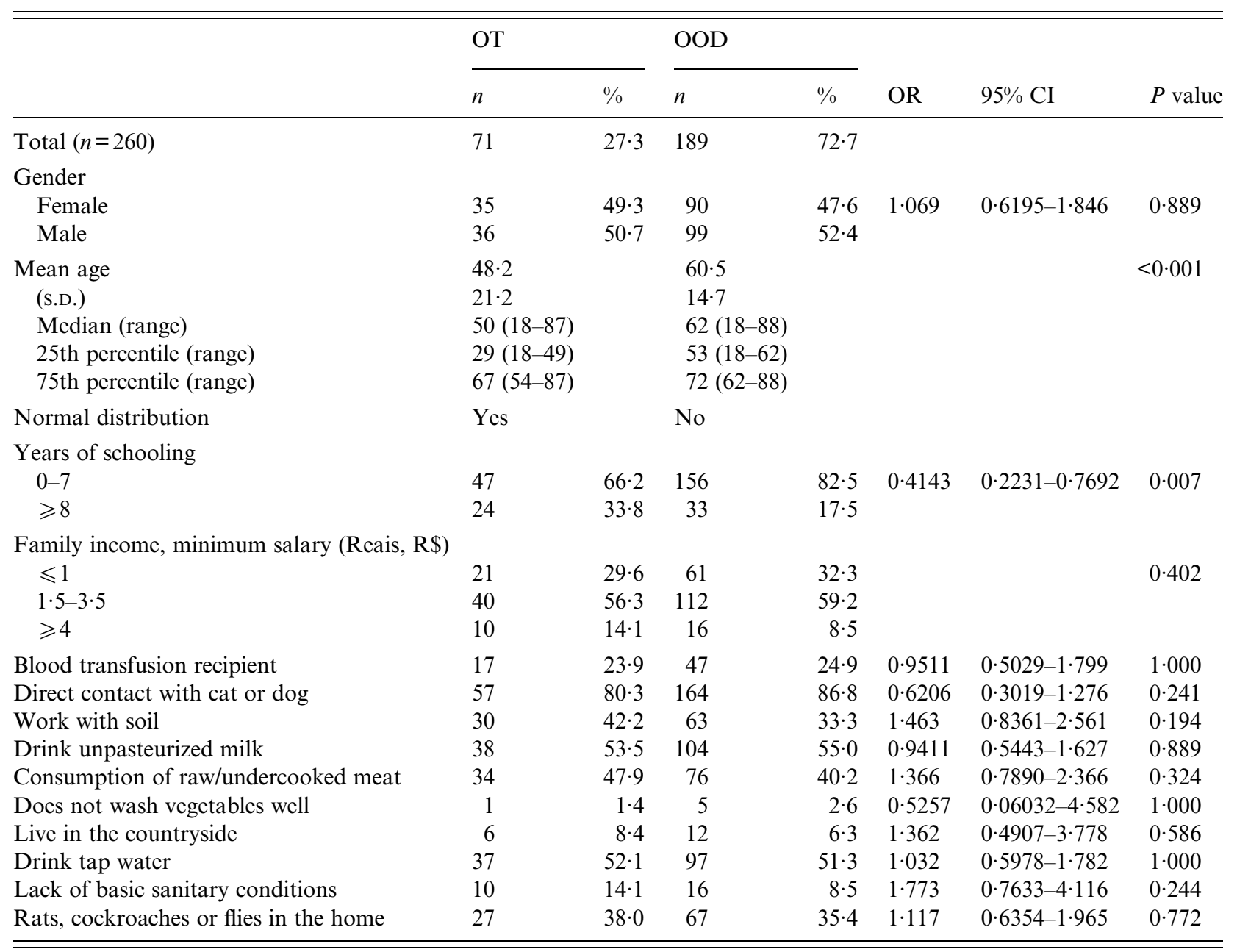

OR, Odds ratio; CI, confidence interval.

* Univariate analysis; $95 \%$ confidence interval, $P \leqslant 0 \cdot 05$.

Aleixo et al. [27] found an association between ocular lesions suggestive of toxoplasmosis and women, while Jones et al. [29] reported a higher prevalence of OT in men, possibly due to greater contact with soil on farms and rural settings. These data suggest that the prevalence of OT by gender may be dependent on environmental and biological risk factors that were not present in our population.

No association was found between $T$. gondii infection and the mean age or the socioeconomic conditions, but the difference in mean age was statistically significant between seropositive patients with OT and those with OOD. Patients with OT had a lower mean age than those with OOD; OT can affect individuals at any stage of life, with $70-90 \%$ of cases being due to congenital infections and $2-30 \%$ due to infections acquired after birth [30].

Additional analysis in OT patients showed lower values for the 25 th percentile regarding age compared to those with OOD. This observation is consistent with previous reports and suggests that the high incidence of eye disease in congenital toxoplasmosis in Brazil causes patients to seek ophthalmology consultations early [3, 17, 23, 31, 32].

These findings could provide some background for public policies towards maternal fetal and neonatal screening in São Paulo state and postnatal monitoring for the early identification and characterization of toxoplasmosis in children, thereby avoiding later complications and delays in the 


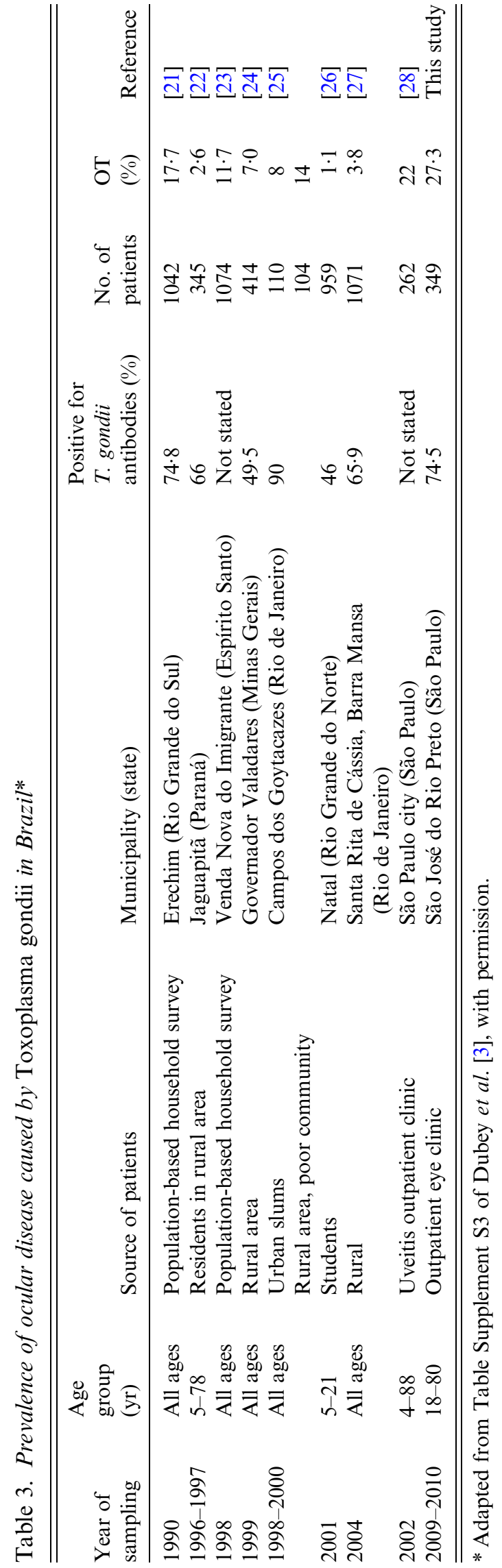

characterization of OT in children, adolescents and young adults.

In a study conducted in the High-Risk Pregnancy Clinic of Hospital de Base in São José do Rio Preto, the rate of congenital transmission in the region proved to be $2 \cdot 3 \%$ [33] and the acute infection rate in 556 pregnant women was $3 \cdot 4 \%$ [18].

However, there is no government programme of maternal fetal and neonatal screening in the São Paulo state nor is there postnatal monitoring for the early identification and characterization of toxoplasmosis in children, which complicates and delays the characterization of OT in children, adolescents and young adults.

There was no association between seropositive and seronegative patients and the level of schooling. A lower percentage of OT was observed in those stating that they were illiterate or had few years of schooling. This finding seems paradoxical since less schooling implies greater exposure to risk due to not adopting appropriate measures, such as good hygiene practice, to prevent infection [34]. It would be expected that individuals with a higher level of schooling would have a lower risk of developing OT. It is possible that individuals with better schooling go to eye clinics more frequently and therefore the diagnosis in this group is higher.

The association observed in this study between infection with $T$. gondii and direct contact with cats and dogs has been reported previously [12, 35]. This observation is plausible because the cat is the definitive host of the parasite and thus eliminates oocysts in stools, which after maturation, contaminate the environment [36]. It is believed that consumption of canine meat may contribute to the spread of the parasite in humans, as has been demonstrated in other diseases [37]; however, Brazilians do not have the habit of eating canine meat. However, there is the possibility of contamination due to oocysts in dog fur, as well explained by Frenkel \& Parker [38].

It was noted in the current study that infection is strongly associated with the consumption of raw or undercooked meat, a habit believed to be the main infection route of $T$. gondii. An association was also found in this study, albeit insignificant, between T. gondii infection and regular contact with soil. Direct contact with earth/soil suggests that infection may also occur in this way [11, 39]; and industrialized processed meat and poor hygiene habits also contribute to infection with $T$. gondii [40-42]. 
No association was found with other factors evaluated and $T$. gondii infection. No statistically significant differences were observed when environmental risk factors associated with $T$. gondii infection were analysed in relation to the development of OT. As the risk factors for infection are not associated with $\mathrm{OT}$, it is possible that the risk of developing OT is dependent on the pathogenicity of the infecting strain and/or the immune competence of the host coupled with immunogenetic factors that determine susceptibility. However, these factors are inherent in patients and the parasite requires further study.

In conclusion, the results of this study confirm that the presence of dogs and cats, as well as the consumption of raw or undercooked meat increases the risk of infection, but does not influence the development of OT.

\section{ACKNOWLEDGEMENTS}

This study was funded by the Fundação de Amparo à Pesquisa do Estado de São Paulo-FAPESP (L.C.M., no. 2009/17540-2) (C.S.M., no. 2009/09168-6) (V.L.P.C., no. 2011/13939-8) (F.N. no. 2012/07750-2) (C.R.B., no. 2012/05580-2); Brazilian Ministry of Science, Technology and Inovation-CNPq (L.C.M., no. 473579/2009-0); Brazilian Ministry of EducationCAPES Scholarship (A.I.C.F, C.C.B.M, C.R.B); and BAP-FAMERP (L.C.M).

The authors thank J.P. Dubey and colleagues, for sharing the data in Table 3, previously published in reference [3]. Thanks are also due to: Dr Lilian Castiglioni (Department of Public Health; FAMERP) for statistical analysis support, and David Hewitt and Jim Henson (AcademicEnglishSolutions.com) for English revision. The authors also thank the Biotechnology Research Institute of Auckland University of Technology, New Zealand, particularly Professor Stephen Henry.

\section{DECLARATION OF INTEREST}

None.

\section{REFERENCES}

1. Hill D, Dubey JP. Toxoplasma gondii: transmission, diagnosis and prevention. Clinical Microbiology and Infection 2002; 8: 634-640.

2. Tenter AM, Heckeroth AR, Weiss LM. Toxoplasma gondii: from animals to humans. International Journal for Parasitology 2000; 30: 1217-1258.
3. Dubey JP, et al. Toxoplasmosis in humans and animals in Brazil: high prevalence, high burden of disease, and epidemiology. Parasitology 2012; 139: 1375-1424.

4. Baldursson S, Karanis P. Waterborne transmission of protozoan parasites: review of worldwide outbreaks an update 2004-2010. Water Research 2011; 45: 6603 6614.

5. Klaren VNA, Kijlstra A. Toxoplasmosis, an overview with emphasis on ocular involvement. Ocular Immunology and Inflammation 2002; 10: 1-26.

6. Antoniazzi E, et al. Ocular impaiment of toxoplasmosis. Parassitologia 2008; 50: 35-36.

7. Weiss LM, Dubey JP. Toxoplasmosis: a history of clinical observations. International Journal for Parasitology 2009; 39: 895-901.

8. Hay J, Dutton GN. Toxoplasma and the eye. British Medical Journal 1995; 310: 1021-1022.

9. Matos K, et al. Correlation between clinical diagnosis and PCR analysis of serum, aqueous, and vitreous samples in patients with inflammatory eye disease. Arquivos Brasileiros de Oftalmologia 2007; 70: 109-114.

10. Arevalo JF, et al. Ocular toxoplasmosis in the developing world. International Ophthalmology Clinics 2010; 50: $57-69$.

11. Spalding SM, et al. Serological screening and toxoplasmosis exposure factors among pregnant women in South of Brazil. Revista da Sociedade Brasileira de Medicina Tropical 2005; 38: 173-177.

12. Barbosa IR, Holanda CMCX, Andrade-Neto VF. Toxoplasmosis screening and risk factors amongst pregnant females in Natal, northeastern Brazil. Transactions of the Royal Society of Tropical Medicine and Hygiene 2009; 103: 377-382.

13. Mattos CCB, et al. Contribution of laboratory methods in diagnosing clinically suspected ocular toxoplasmosis in Brazilian patients. Diagnostic Microbiology and Infectious Disease 2011; 70: 362-366.

14. Kapperud G, et al. Risk factors for Toxoplasma gondii infection in pregnancy. American Journal of Epidemiology 1996; 144: 405-412.

15. Avelino MM, et al. Risk factors for Toxoplasma gondii infection in women of childbearing age. Brazilian Journal of Infectious Disease 2004; 8: 164-174.

16. Boyer KM, et al. Risk factors for Toxoplasma gondii infection in mothers of infants with congenital toxoplasmosis: implications for prenatal management and screening. American Journal of Obstetrics and Gynecology 2005; 192: 564-571.

17. Robert-Gangneux F, Dardé ML. Epidemiology of and diagnostic strategies for toxoplasmosis. Clinical Microbiology Review 2012; 25: 264.

18. Gonçalves MAS, et al. Seropositivity rates for toxoplasmosis, rubella, syphilis, cytomegalovirus, hepatitis and HIV among pregnant women receiving care at a Public Health Service, São Paulo State, Brazil. Brazilian Journal of Infectious Disease 2010; 14: 427432.

19. Mattos CCB, et al. Lack of association between ABO histo-blood groups, secretor and non-secretor phenotypes, and anti-Toxoplasma gondii antibodies among 
pregnant women from the northwestern region of São Paulo State, Brazil. Archives of Medical Science 2008; 4: 254-258.

20. Rodrigues ACF, et al. Non-association between antiToxoplasma gondii antibodies and ABO blood group system. Journal of Venomous Animals and Toxins including Tropical Diseases 2011; 17: 184-189.

21. Glasner PD, et al. An unusually high prevalence of ocular toxoplasmosis in southern Brazil. American Journal of Ophthalmology 1992; 114: 136-144.

22. Garcia JL, et al. Seroprevalence, epidemiology, and ocular evaluation of human toxoplasmosis in a rural area in Jaguapitã, Paraná, Brazil. Revista Panamericana de Salud Pública 1999; 6: 157-163.

23. Abreu MT, et al. Ocular toxoplasmosis at Venda Nova do Imigrante, ES, Brasil. Arquivos Brasileiros de Oftalmologia 1998; 61: 540-545.

24. Portela RWD et al. A multihousehold study reveals a positive correlation between age, severity of ocular toxoplasmosis, and levels of glycoinositolphospholipidspecific immunoglobulin A. Journal of Infectious Diseases 2004; 190: 175-183.

25. Bahia-Oliveira LMG, et al. Toxoplasmosis in southeastern Brazil: an alarming situation of highly endemic acquired and congenital infection. In: Petersen E, Pollak A, Owona IR, eds. Recent Trends in Research on Congenital Toxoplasmosis. International Journal for Parasitology 2001; 31: 133-136.

26. Amorim GCA, et al. Socioeconomic conditions as determining factors in the prevalence of systemic and ocular toxoplasmosis in northeastern Brazil. Ophthalmic Epidemiology 2004; 11: 301-317.

27. Aleixo ALQC, et al. Frequency of lesions suggestive of ocular toxoplasmosis among a rural population in the State of Rio de Janeiro. Revista da Sociedade Brasileira de Medicina Tropical 2009; 42: 165-169.

28. Gouveia EB, et al. Causes of uveitis in a tertiary centre in São Paulo city, Brazil. Arquivos Brasileiros Oftalmologia 2004; 67: 139-145.

29. Jones JL, et al. Recently acquired Toxoplasma gondii Infection, Brazil. Emerging Infectious Diseases 2006; 12: $582-587$.

30. Furtado JM, et al. Ocular toxoplasmosis I: parasitology, epidemiology and public health. Clinical and Experi- mental Ophthalmology. Published online 11 July 2012. doi: 10.1111/j.1442-9071.2012.02821.x

31. Joynson DHM, Wreighitt TG. Toxoplasmosis-A Comprehensive Clinical Guide. Cambridge University Press, 2005, pp. 1-395.

32. Latkany P. Ocular disease due to Toxoplasma gondii. In: Weiss LM, Kim K, eds. Toxoplasma gondii: The Model Apicomplexan: Perspectives and Methods. Elsevier/Academic Press: Amsterdam, 2007, pp. $1-17$.

33. Mattos CCB et al. Anti-Toxoplasma gondii antibodies in pregnant women and their newborn infants in the region of São José do Rio Preto, São Paulo, Brazil. Sao Paulo Medical Journal 2011; 129: 261-266.

34. Varella IS, et al. Seroprevalence of toxoplasmosis in pregnant women. Jornal Pediatria 2003; 79: 69-74.

35. Lopes FMR, et al. Factors associated with seropositivity for anti-Toxoplasma gondii antibodies in pregnant women of Londrina, Paraná, Brazil. Memórias do Instituto Oswaldo Cruz 2009; 104: 378-382.

36. Dubey JP, Lindsay DS, Speer CA. Structures of Toxoplasma gondii tachyzoites, bradyzoites, and sporozoites and biology and development of tissue cysts. Clinical Microbiology Review 1998; 11: 267-299.

37. Wertheim HFL, et al. Furious rabies after an atypical exposure. PLoS Medicine 2009; 6: e1000044.

38. Frenkel JK, Parker BB. An apparent role of dogs in the transmission of Toxoplasma gondii: the probable importance of xenosmophilia. Annals of the New York Academy of Science 1996; 791: 402-407.

39. Cook AJC, et al. Sources of toxoplasma infection in pregnant women: European multicentre case-control study. British Medical Journal 2000, 321: 142-147.

40. Bojar I, Szymanska J. Enviromental exposure of pregnant women to infection with Toxoplasma gondiistate of the art. Annals of Agricultural and Environmental Medicine 2010; 17: 209-214.

41. Mecca JN, Meireles LR, Andrade Jr. HF. Quality control of Toxoplasma gondii in meat packages: standardization of an ELISA test and its use for detection in rabbit meat cuts. Meat Science 2011; 88: 584-589.

42. Alvarado-Esquivel C, et al. Toxoplasma gondii infection in workers occupationally exposed to raw meat. Occupational Medicine 2011; 61: 265-269. 